\title{
ON UNIVERSAL NULL SETS
}

\section{E. GRZEGOREK AND C. RYLL-NARDZEWSKI}

\begin{abstract}
If all subsets of cardinality less than $2^{\boldsymbol{t}_{0}}$ of the real line $R$ are Lebesgue measurable then there exists a permutation $p$ of $R$ with $p=p^{-1}$ such that on the $\sigma$-field generated by $\mathscr{B} \cup p(\mathscr{B})$ there is no continuous probability measure.
\end{abstract}

1. Let $|S|$ denote the cardinality of the set $S$. If $f$ is a function from a set $S$ into a set $T$ and $\mathscr{F}$ is a family of subsets of $S$ then by $f(\mathscr{F})$ we denote the family $\{f(F): F \in \mathcal{F}\}$ of subsets of $T$. Let $\mathcal{C}$ be a $\sigma$-field on $S$ (i.e. a countably additive algebra of subsets of $S$ ). If $X \subset S$ then $\subset \cap X$ will denote the $\sigma$-field $\{C \cap X: C$ $\in \mathcal{C}\}$ on $X$. A countably additive measure $\mu$ on a countably generated $\sigma$-field $\mathcal{C}$ on $S$ will be called a nontrivial continuous measure iff $0<\mu(S)<\infty$ and $\mu$ vanishes for all atoms of $\mathcal{C}$. We will say that $\mathcal{C}$ is measurable if there exists a nontrivial

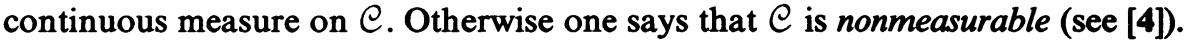

Recall that a subset $Y$ of a separable metrizable space $X$ is called a universal null set iff for every nontrivial continuous measure $\mu$ on $\mathscr{B}_{X}$ (= Borel subsets of $X$ ) we have $\mu^{*}(Y)=0$, where $\mu^{*}$ is the outer measure induced by $\mu$ (see [1], [7] and [13]).

It is easy to check that $Y$ is a universal null set iff $\mathscr{B}_{Y}$ is a nonmeasurable $\sigma$-field on $Y$ (see [11] and [13]). Marczewski and Sierpiński discovered in [11] an uncountable universal null subset of the real line. Further information on universal null sets can be found e.g. in [1], [2], [4], [6], [7], [11] and [13], where there are also other references. A separable metric space $X$ is called absolute Borel if $X$ is a Borel subset of its completion.

2. We work in ZFC set theory.

If $X$ is a separable metrizable space, then we shall consider the following conditions concerning $X$.

(i) There exists a universal null subset $Y$ of $X$ with $|Y|=|X|$.

(ii) There exists a permutation $p$ of $X$ with $p=p^{-1}$ and such that the graph of $p$ is a universal null subset of $X \times X$.

(iii) There exists a permutation $p$ of $X$ with $p=p^{-1}$ and such that the $\sigma$-field generated by $\mathscr{B}_{X} \cup p\left(\mathscr{B}_{X}\right)$ is nonmeasurable.

In connection with (i) it is worth mentioning that for $X=R$ condition (i) is a theorem of ZFC + all subsets of $R$ of cardinality less than $2^{\mu_{0}}$ are Lebesgue

Received by the editors December 15, 1978 and, in revised form, March 31, 1980.

1980 Mathematics Subject Classification. Primary 04-00, 04A15, 04A05; Secondary 28A05, 28A60, 28A10, 28A20, 28A35.

Key words and phrases. $\sigma$-field of sets, continuous measure, Lebesgue measure, universal null set, universally measurable set, Borel set, universally measurable function, bimeasurable function, continuum hypothesis, Martin's Axiom. 
measurable [4], but (i) is independent of ZFC $+2^{k_{0}}>\aleph_{1}$. More precisely, Laver [6, p. 152] has,announced the existence of a model for ZFC $+2^{\varkappa_{0}}>x_{1}$ in which there are no universal null subsets of $R$ of power greater than $\aleph_{1}$. It is known that Martin's Axiom (MA) implies that all subsets of $R$ of cardinality $<2^{\mu_{0}}$ are Lebesgue measurable; moreover $\mathrm{ZFC}+\mathrm{MA}+2^{\boldsymbol{x}_{0}}>\boldsymbol{\aleph}_{1}$ is consistent provided ZFC is consistent. More references can be found in [6] and [8].

The aim of this note is to prove the following

THEOREM. (a) (ii) if and only if (iii).

(b) (i) implies (iii).

(c) If $X$ contains an uncountable absolute Borel subset then (ii) implies (i).

(d) There exists a set $X \subset R$ of positive outer Lebesgue measure such that (i) holds for $X$.

The following corollaries are strengthenings of Theorems 1 and 2 from [4].

COROLlaRy 1. There exist a countably generated and separating points measurable $\sigma$-field $\mathcal{C}$ on a set $S$ and a permutation $p$ of $S$ with $p=p^{-1}$ and such that the $\sigma$-field generated by $\mathcal{\cup} \cup(\mathcal{C})$ is nonmeasurable.

COROLlARY 2. There exist a countably generated measurable $\sigma$-field $\mathcal{C}$ on $R$ and $a$ permutation $p$ of $R$ with $p=p^{-1}$ and such that the o-field generated by $\mathcal{P} \cup(\mathcal{C})$ is nonmeasurable.

We do not know the answer to the following questions.

(1) Let $X$ be a nonempty subset of $R$ such that each subset $Y$ of $X$ with $|Y|=|X|$ has positive outer Lebesgue measure (the existence of such sets has been proved by Sierpiński (see e.g. [10]) under hypothesis $2^{\kappa_{0}}=\aleph_{1}$, in fact MA is sufficient). It is evident that all such $X$ 's do not have property (i). Does $X$ have property (iii)?

(2) Does there exist a subset $X$ of $R$ such that (iii) holds but (i) fails?

3. We start with a proof of the theorem. Part (a) follows easily from the following

LEMMA. Let $\bigodot$ be a $\sigma$-field on a set $S$, let $p$ be a permutation of $S$ and let $P \subset S \times S$ be the graph of $p$. Then the $\sigma$-field $\mathscr{D}$ on $S$ generated by $\mathcal{\cup} \cup(\mathcal{C})$ is

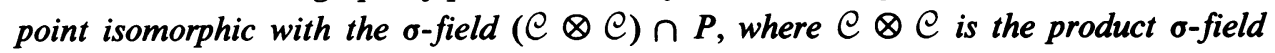
on $S \times S$.

Proof of the Lemma. Consider the projection map $\pi: P \rightarrow S, \pi(x, y)=y$ where $(x, y) \in P$. Since $p$ is a permutation, $\pi$ is a one-to-one map from $P$ onto $S$. For every $C \in \mathcal{C}$ we have $\pi((C \times S) \cap P)=p(C)$ and $\pi((S \times C) \cap P)=C$; conse-

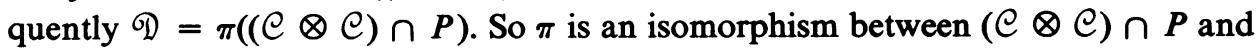
(2).

Let $p$ be a permutation of a separable metrizable space $X$ and let $P \subset X \times X$ be the graph of $p$. Since $\left(\mathscr{B}_{X} \otimes \mathscr{G}_{X}\right) \cap P=\mathscr{B}_{P}$, by the lemma we have that the $\sigma$-field $\mathscr{B}_{P}$ is nonmeasurable if and only if the $\sigma$-field generated by $\mathscr{B}_{X} \cup p\left(\mathscr{G}_{X}\right)$ is nonmeasurable. Hence conditions (ii) and (iii) are mutually equivalent. 
Proof of (b). Assume that $X$ satisfies (i). For finite $X$ (iii) is obvious; assume that $X$ is infinite. Hence there exists a universal null subset $Y$ of $X$ with $|Y|=$ $|X \backslash Y|$. Let $p$ be a permutation of $X$ such that $p=p^{-1}$ and $p(Y)=X \backslash Y$. We claim that $p$ satisfies (iii). If not, let $\mu$ be a nontrivial continuous measure on the $\sigma$-field generated by $\mathscr{B}_{X} \cup p\left(\mathscr{B}_{X}\right)$. Let $\mu_{1}$ be the restriction of $\mu$ to $\mathscr{B}_{X}$ and let $\mu_{2}$ be the restriction of $\mu$ to the $\sigma$-field $p\left(\mathscr{B}_{X}\right)$. Define a nontrivial continuous measure $\nu$ on $\mathscr{B}_{X}$ putting $\nu(B)=\mu_{2}(p(B))$ for all $B \in \mathscr{B}_{X}$. Since $Y$ is a universal null subset of $X$ we have $\mu_{1}^{*}(Y)=\nu^{*}(Y)=0$; moreover $\nu^{*}(Y)=\mu_{2}^{*}(p(Y))=\mu_{2}^{*}(X \backslash Y)$. So

$$
\mu^{*}(X)<\mu^{*}(Y)+\mu^{*}(X \backslash Y)<\mu_{1}^{*}(Y)+\mu_{2}^{*}(X \backslash Y)=0 ;
$$

hence we get a contradiction.

Proof of (c). Assume that $X$ satisfies (ii). Applying Marczewski's theorem (see [12, p. 144]) we can obtain a subset $Z$ of $R$ such that the $\sigma$-field $\mathscr{B}_{X \times X} \cap$ (graph $p$ ) is isomorphic with the $\sigma$-field $\mathscr{B}_{Z}$. Let $B$ be an uncountable absolute Borel subset of $X$. It is well known that $\mathscr{B}_{B}$ is isomorphic to $\mathscr{B}_{R}$ (see e.g. [5, p. 451]). Hence there exists a subset $Y$ of $B$ such that $\mathscr{B}_{X \times X} \cap\left(\right.$ graph $p$ ) is isomorphic with $\mathscr{B}_{Y}$. Since graph $p$ is a universal null subset of $X \times X$ we have that (graph $p$ ) $\cap \mathscr{B}_{X \times X}$ is a nonmeasurable $\sigma$-field and hence $\mathscr{B}_{Y}$ is a nonmeasurable $\sigma$-field on $Y$. Therefore $Y$ is a universal null subset of $X$ and $|Y|=\mid$ graph $p|=| X \mid$.

Proof of (d). There exist (see Corollary 2 in [4]) subsets $A$ and $B$ of $R$ such that $|A|=|B|, A$ is a universal null set (called in [4] absolutely of measure zero) and $B$ has positive outer Lebesgue measure. Put $X=A \cup B$. It is evident that $X$ has property (i).

Proof of Corollary 1. Let $X$ be as in the theorem, part (d). By part (b) there exists a permutation $p$ of $X$ satisfying (iii). Put $\mathcal{C}=\mathscr{B}_{X}$ and $S=X$. Since outer Lebesgue measure restricted to $\mathscr{B}_{X}$ is a nontrivial continuous measure on $\mathscr{B}_{X}$ we have that $\mathcal{C}$ is a measurable $\sigma$-field as was required.

Proof of Corollary 2. Let $X$ and $p$ be as in the theorem, part (d). Let $\mathcal{C}$ be the $\sigma$-field on $R$ generated by $\mathscr{B}_{X} \cup\{R \backslash X\}$. Since, as it was observed in the proof of Corollary $1, \mathscr{B}_{X}$ is measurable we have that $\mathcal{C}$ is a countably generated measurable $\sigma$-field on $R$. Let $p^{\prime}$ be a permutation of $R$ defined by $p^{\prime}(x)=p(x)$ for all $x \in X$, and $p^{\prime}(x)=x$ for all $x \in R \backslash X$. It is easy to check that $p^{\prime}$ and $\mathcal{C}$ have the required properties.

4. If $X$ and $Y$ are separable metrizable spaces such that $|X|=|Y|$, then we shall consider the following conditions concerning $X$ and $Y$.

(i') There exist universal null subsets $X_{1}$ of $X$ and $Y_{1}$ of $Y$ with $\left|X_{1}\right|=|X|$ and $\left|Y_{1}\right|=|Y|$.

(ii') There exists a one-to-one function $p$ from $X$ onto $Y$ such that the graph of $p$ is a universal null subset of $X \times Y$.

(iii') There exists a one-to-one function $p$ from $X$ onto $Y$ such that the $\sigma$-field generated by $\mathscr{B}_{Y} \cup p\left(B_{X}\right)$ is nonmeasurable.

Recall that a subset $X_{1}$ of a separable metrizable space $X$ is called universally measurable iff for every probability measure $\mu$ on $\mathscr{B}_{X}$ there exist $B_{1}, B_{2} \in \mathscr{B}_{\text {such }}$ that $B_{1} \subset X \subset B_{2}$ and $\mu\left(B_{2} \backslash B_{1}\right)=0$ (see e.g. [7]). 
A proof of the following Theorem' is an easy modification of the proof of the theorem, and will be omitted.

THEOREM' . (a) (ii') if and only if (iii').

(b) (i') implies (iii').

(c) If $X$ and $Y$ contain uncountable absolute Borel sets then (ii') implies (i').

(d) If $B$ is an uncountable absolute Borel set then there exists a subset $X$ of $B$ such that $X$ is not universally measurable and there exists a universal null subset $X_{1}$ of $X$ with $\left|X_{1}\right|=|X|$.

The following Corollary 3 will be useful.

Corollary 3. Let $K$ and $C$ be uncountable absolute Borel sets. Then there exists a universal null subset $N$ of $K \times C$ such that the projections of $N$ onto $K$ and onto $C$ are not universally measurable subsets of $K$ and $C$, respectively.

Proof of Corollary 3. By part (d) of Theorem' there exists a subset $X$ of $K$ such that $X$ is not universally measurable and there exist a universal null subset $X_{1}$ of $X$ with $\left|X_{1}\right|=|X|$. Let $f$ be a Borel isomorphism between $K$ and $C$. Put $Y=f(X)$ and $Y_{1}=f\left(X_{1}\right)$. It is easy to see that $Y$ is a subset of $C$ which is not universally measurable, $Y_{1}$ is a universal null subset of $Y$ and $\left|Y_{1}\right|=|Y|=|X|$. By Theorem' we have that (i') implies (ii'). Hence there exists a one-to-one function $p$ from $X$ onto $Y$ such that the graph of $p, N$, is a universal null subset of $X \times Y$. It is evident that $N$ has the properties required in Corollary 3.

Corollary 3 permits the elimination of MA from the assumption of the theorem of Darst [3], that a Borel function $f$, mapping a Borel subset, $D_{f}$, of a separable complete metric space, $M_{1}$, into a separable complete metric space, $M_{2}$, maps Borel subsets of $D_{f}$ onto Borel subsets of $M_{2}$ if and only if $f$ maps universally measurable subsets of $D_{f}$ onto universally measurable subsets of $M_{2}$.

Darst has proved his theorem with the help of the continuum hypothesis and has written [3, p. 566]: "We shall need to employ the continuum hypothesis only in the last step of our argument."

The mentioned last step of the proof of Darst is a part of Corollary 3. Mauldin has proved that the continuum hypothesis can be replaced there by MA [9, p. 60-61].

REMARK 1. The implication that (i') imples (ii') can also be proved easily directly.

RemARK 2 (ADDED MARCH 15, 1980). In 1979 G. V. Cox ( $A$ universal null graph whose domain has positive measure, Colloq. Math. (to appear)), assuming the continuum hypothesis, answered both our questions. Namely he proved, in ZFC + $\mathrm{CH}$, the existence of two uncountable subsets $X_{1}, X_{2}$ of $R$ such that each uncountable subset of $X_{1}$ and each uncountable subset of $X_{2}$ have positive outer Lebesgue measure, (ii) holds for $X_{1}$ and (ii) does not hold for $X_{2}$.

\section{REFERENCES}

1. S. Banach, Sur les suites d'ensembles excluant l'existence d'une mesure (Note posthume avec préface et commentaire de E. Marczewski), Colloq. Math. 1 (1948), 103-108.

2. S. Braun and E. Szpilrajn-Marczewski (en collaboration avec C. Kuratowski), Annexe, Fund. Math. 1 (1937), 225-254. 
3. R. B. Darst, A characterizatkon of bimeasurable functions in terms of universally measurable sets, Proc. Amer. Math. Soc. 27 (1971), 566-571.

4. E. Grzegorek, Solution of a problem of Banach on o-fields without continuous measures, Bull. Acad. Polon. Sci. Sér. Sci. Math. (1979/1980) (to appear).

5. C. Kuratowski, Topology. I, Academic Press, New York; PWN, Warsaw, 1966.

6. R. Laver, On the consistency of Borel's conjecture, Acta Math. 137 (1976), 151-169.

7. E. Marczewski, Remarque sur la measurabilité absolute, Colloq. Math. 1 (1948), 42-43.

8. D. A. Martin and R. M. Soloway, Internal Cohen extensions, Ann. Math. Logic 2 (1970), 143-148.

9. R. D. Mauldin, Some effects of set-theoretical assumption in measure theory, Advances in Math. 27 (1978), 45-62.

10. J. C. Oxtoby, Measure and category, Springer-Verlag, Berlin and New York, 1971.

11. W. Sierpinski and E. Szpilrajn-Marczewski, Remarque sur le probleme de la mesure, Fund. Math. 26 (1936), 256-261.

12. E. Szpilrajn-Marczewski, On the isomorphism and the equivalence of classes and sequences of sets, Fund. Math. 32 (1939), 133-148.

13. __ Sur les ensembles et les fonctions absolument mesurables, C. R. Soc. Sci. Lett. Varsovie, Cl. III 30 (1937), 39-68 (Polish). (An English translation, by John C. Morgan II, of this article is available in manuscript.)

Institute of Mathematics, Polish Academy of Sciences, 51-617 Wrockaw, Poland

Institute of Mathematics, Techinical University, 50-370 Wrockaw, Poland 